\title{
Tuberculosis en individuos con infección por VIH en Chile: Estudio de prevalencia e impacto sobre mortalidad
}

\author{
Luis Villarroel $^{\text {la, }}$, Ricardo Rabagliati², M. Elvira Balcells ${ }^{2}$, \\ Lorena Karzulovic ${ }^{3 b}$, Carlos Pérez ${ }^{2}$. \\ Prevalence of tuberculosis \\ and its impact on mortality \\ among HIV infected patients in Chile
}

Background: Tuberculosis (TB) in Chile is reaching the elimination phase; however, in HIV positive individuals the incidence of TB in still very high. Aim: To describe the association between TB and HIV in different geographical regions in Chile, and to determine the association between TB and HIV/AIDS mortality. Patients and methods: A retrospective study that included individuals from the main HIV clinics from four regions with different TB prevalence in the general population (per 100,000): Arica (>30), Concepcion/Arauco (25-29), Valparaíso/San Antonio $(20-24)$ and Metropolitana Sur-Oriente (SSMSO) $(<20)$, attended between january 1998 and september 2004. Results: Nine hundred and twelve HIV positive individuals were included. Gobal prevalence of TB was 6.2\% [95\% confidence intervals (CI) 5.2-7.2\%]. TB was more common in older subjects ( $p=0.039$ ) and those with lower CD4 counts ( $p \varangle 0.001$ ) and higher HIV viral load ( $p=0.033$ ). In $66 \%$ of cases, the disease had a pulmonary localization. TB was the recorded cause of death in $7.4 \%$ of subjects. Only $29 \%$ of patients had a tuberculin skin test performed at the moment of HIV diagnosis. The prevalence of TB in HIV positive patients, followed the trend of TB prevalence in the general population: Concepcion/Arauco (11.9\%), Valparaiso/San Antonio (7.1\%) and SSMSO (3.9\%). However HIV positive subjects from Arica showed an unexpectedly low TB prevalence (5.5\%). Conclusions: TB in HIV/AIDS patients included in this study is over 300 times more prevalent than in the general population. TB prevalence in HIV positive subjects follows regional TB prevalence, excepting Arica. Effectiveness and feasibility of latent TB diagnostic strategies and treatment in HIV positive individuals should be reviewed (Rev Méd Chile 2008; 136: 578-86).

(Key words: HIV; Prevalence; Tuberculosis)

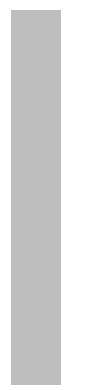

\begin{abstract}
Recibido el 23 de agosto, 2007. Aceptado el 24 de enero, 2008.
Proyecto colaborativo entre la Comisión Nacional del Sida y la Pontificia Universidad Católica de Chile, en el marco del Proyecto "Aceleración y profundización de la respuesta nacional, intersectorial, participativa y descentralizada a la epidemia del VIH/SIDA en Chile", del Proyecto Fondo Global Chile. Esta instancia tuvo influencia en el diseño del estudio. La recolección, análisis e interpretación de los datos fue de responsabilidad de los autores.

${ }^{1}$ Departamento de Salud Pública, ${ }^{2}$ Programa de Enfermedades Infecciosas, Departamento de Medicina Interna, Facultad de Medicina, ${ }^{3}$ Escuela de Medicina, Pontificia Universidad Católica de Chile. Santiago de Chile.

aDoctor en Estadística.

bEstudiante de Medicina, Pontificia Universidad Católica de Chile.
\end{abstract}

Correspondencia a: Dr. Luis Villarroel del Pino. Departamento de Salud Pública, Facultad de Medicina, Pontificia Universidad Católica de Chile. Marcoleta 434. Santiago. Fax: 633-1840.

E mail: lv@med.puc.cl 
$\mathrm{E}^{\mathrm{n}}$ n 1993, la Organización Mundial de la Salud (OMS) declaró a la tuberculosis (TBC) "Emergencia Global" debido a su importante resurgimiento, implementando diversas medidas estratégicas de control global. Pese a ello, el año 2005 se estimaron 8,8 millones de casos nuevos a nivel mundial, llevando a una prevalencia de 14 millones de individuos enfermos de $\mathrm{TBC}^{1}$.

Diversos factores han contribuido a la reemergencia de TBC tales como el aumento de las migraciones, dificultades en el diagnóstico y tratamiento en países de escasos recursos, la aparición de cepas resistentes e importantemente la creciente pandemia de $\mathrm{VIH}$. A nivel mundial, el número estimado de personas viviendo con VIH (PVVIH) es de 39,5 millones, con una incidencia anual de 4,3 millones ${ }^{2}$. Como resultado de la interacción entre TBC y VIH se genera una epidemia dual, ya que la infección por VIH es un factor de riesgo tanto para primoinfección TBC como para progresión más rápida a enfermedad TBC. Las cifras globales de asociación TBC-VIH demuestran que $11 \%$ de los casos nuevos de TBC y $12 \%$ de muertes por TBC en adultos son atribuibles a la infección por $\mathrm{VIH}^{3}$.

En Chile, la tasa de morbilidad TBC reportada en 2003 fue de 18,4 por 100.000 habitantes, lo que sitúa al país en una fase epidemiológica de eliminación de la enfermedad ${ }^{4}$. Sin embargo, la magnitud de la morbilidad por TBC es muy variable en las distintas zonas geográficas del país. El Servicio de Salud de Arica es el que tiene la mayor tasa de TBC $(38,2 / 100.000$ habitantes) y la tasa más baja se encuentra en el Servicio de Salud de San Felipe $(3,4 / 100.000)^{4}$. Por otra parte, hasta 2005 se habían notificado 15.894 casos de VIH/SIDA, distribuidos desigualmente a lo largo del país. La Región de Tarapacá mostraba la tasa acumulada más alta de notificación de VIH (94/100.000) seguida por la Región Metropolitana $(87 / 100.000)^{5}$. Según datos nacionales la prevalencia de TBC sería al menos 100 veces mayor en PVVIH que en el resto de la población ${ }^{6}$. Sin embargo, a nuestro conocimiento no existen datos publicados de las diferencias regionales en las tasas de TBC en individuos VIH positivos.

El objetivo principal del presente estudio fue caracterizar la asociación TBC/VIH en Chile en general y específicamente por zonas geográficas y como objetivo secundario evaluar el impacto de la TBC en la mortalidad por VIH/SIDA.

\section{MATERIAL Y MÉTODO}

Estudio retrospectivo, basado en la revisión de fichas clínicas de individuos VIH positivos, atendidos en el sistema público de salud en cuatro zonas geográficas, correspondientes a los Centros de Atención de los Servicios de Salud de Arica, Valparaíso/San Antonio, Concepción y Región Metropolitana Sur-Oriente (SSMSO), entre 1 de enero de 1998 y 1 de septiembre de 2004. Estas áreas fueron elegidas por sus diferencias en las tasas poblacionales de morbilidad TBC (Tabla 1).

Una enfermera del equipo de investigación recolectó las variables de interés desde las fichas clínicas, registrando en una base de datos estandarizada. Los datos faltantes fueron complementados con registros de notificaciones a la Comisión Nacional del SIDA (CONASIDA). Se incluyeron edad, género, nacionalidad, nivel educacional, recuento de linfocitos $\mathrm{CD} 4$ basal (CD4), carga viral VIH basal (CV), uso de terapia antirretroviral (TAR), PPD, uso de isoniacida (adecuado/inadecuado según Programa Nacional de Control de la Tuberculosis $^{4}$ ) e historia de enfermedad TBC (fecha, método diagnóstico y localización). En los pacientes fallecidos se consignó la causa de muerte.

Definiciones de enfermedad TBC y TBC latente. Se consideraron las categorías sano TBC, infección

\section{Tabla 1. Tasa de morbilidad T BC en población general (año 2002) en las cuatro zonas geográficas incluidas}

\begin{tabular}{|lr|}
\hline Zona geográfica & $\begin{array}{r}\text { Tasa poblacional } \\
\text { morbilidad TBC } \\
\text { (x100.000 habs.) }\end{array}$ \\
\hline Arica & $>30$ \\
Valparaíso/San Antonio & $20-24$ \\
Metropolitana Sur-Oriente & 20 \\
Concepción/Arauco & $25-29$ \\
Total & 18,4 \\
\hline
\end{tabular}


TBC y enfermedad TBC, según el criterio descrito en el Programa Nacional de Control de la Tuberculosis $^{4}$, que considera infección TBC (o infección latente TBC) en PVVIH aquellos pacientes con PPD $\geq 5 \mathrm{~mm}$ sin evidencias de infección activa por M tuberculosis y enfermedad TBC según la positividad de la evidencia existente (histológica 0 bacteriológica).

Estadística. El marco poblacional está compuesto por 1.661 personas, que corresponde al total de personas atendidas en los 4 centros en el período del estudio (1.381 vivos y 280 fallecidos). El tamaño muestral calculado fue de 912 casos (723 vivos y 189 fallecidos), que permite estimar prevalencias por centro y globales con un margen de error de 4,5 puntos y de 2 a 3 puntos porcentuales con confianza 95\%, respectivamente. La selección de pacientes se realizó en forma aleatoria, proporcional al número de pacientes atendidos en cada centro y según la proporción de individuos vivos y fallecidos (Tabla 2). Para comparación de porcentajes se utilizó test chicuadrado y para comparar promedios se utilizó test $\mathrm{t}$ de Student para muestras independientes. Las tasas de prevalencia y totales poblacionales con enfermedad TBC se estimaron utilizando factores de expansión ${ }^{7,8}$ y se presentan con intervalos de confianza de 95\%. Para evaluar el efecto de la enfermedad TBC sobre la mortalidad se utilizó razón de chances (OR), con intervalo de confianza de 95\%. Para identificar variables asociadas en forma conjunta e independiente con la muerte de los pacientes, y para observar el efecto de la enfermedad TBC, se ajustaron modelos de regresión logística binaria paso-a-paso ${ }^{9}$. Mediante los mismos modelos se estimó OR ajustada, con intervalos de confianza de 95\%. Se consideró significativo todo valor $p<0,05$. Los análisis estadísticos fueron hechos con los programas $\mathrm{R}^{10}$ y SPSS 14.

\section{RESUlTADOS}

Las características epidemiológicas de los 912 individuos incluidos se describen en la Tabla 3. Se identificaron 62 casos de enfermedad TBC (6,8\%), 26 correspondían a individuos fallecidos. La prevalencia global de enfermedad TBC ajustada por sexo, edad y estado del paciente (vivo o fallecido), usando factores de expansión, se estimó en 6,2\% (IC 95\%: 5,2\%-7,2\%).

El método diagnóstico de enfermedad TBC, consignado en 51 pacientes, fue baciloscopia 0 cultivo de Koch (70,6\%), clínico/radiológico $(9,8 \%)$, biopsia $(7,8 \%)$ y otros $(11,8 \%)$. Las localizaciones más frecuentes fueron: pulmonar (66,1\%), ganglionar $(11,3 \%)$ e intestinal $(4,8 \%)$.

En el análisis univariado (Tabla 4), se observó mayor prevalencia de TBC a mayor edad, en hombres, con menor recuento de CD4 y mayor CV. Si bien los pacientes con enfermedad TBC recibieron TAR más frecuentemente, la mayoría $(51,6 \%)$ correspondió a inicio después del diagnóstico de TBC y en 11,3\% fue la enfermedad TBC la indicación de inicio de TAR. Cabe señalar que en $14,5 \%$ de los casos la TBC se diagnosticó luego de haber iniciado la TAR.

La prevalencia de enfermedad TBC por zona geográfica se muestra en Tabla 5 . La significativa mayor prevalencia de TBC en

Tabla 2. Población VIH registrada por centro y tamaños muestrales calculados

\begin{tabular}{|lrrrrrr|}
\hline Zona geográfica & \multicolumn{3}{c}{ Población VIH registrada } & \multicolumn{3}{c|}{ Tamaño muestral } \\
& Vivos & Fallecidos & Total & Vivos & Fallecidos & Total \\
\hline Arica & 165 & 37 & 202 & 117 & 27 & 144 \\
Valparaíso/San Antonio & 285 & 82 & 367 & 169 & 52 & 221 \\
Metropolitana Sur-Oriente & 710 & 104 & 814 & 286 & 74 & 360 \\
Concepción/Arauco & 221 & 57 & 278 & 151 & 36 & 187 \\
Total & 1.381 & 280 & 1.661 & 723 & 189 & 912 \\
\hline
\end{tabular}


Tabla 3. C aracterísticas generales de los 912 individuos con infección por VIH incluidos

\begin{tabular}{|lcc|}
\hline Variable & \multicolumn{2}{c|}{ Promedio \pm DS/n (\%) } \\
\hline Edad (años) & \multicolumn{2}{c|}{$37,8 \pm 11,1$} \\
Sexo femenino & 151 & $(16,6)$ \\
Nacionalidad chilena & 907 & $(99,5)$ \\
Nivel educacional básico o ninguno & 266 & $(30,2)$ \\
Vacunación BCG* & 95 & $(95 \%)$ \\
Enfermedad TBC & 62 & $(6,8)$ \\
CD4 basal (cel/mm ${ }^{3}$ ) & $254 \pm 221$ \\
Logaritmo de Carga Viral basal (copias/ml) & $4,67 \pm 0,96$ \\
Pacientes con indicación de TAR & 506 & $(56,2)$ \\
Pacientes reciben alguna vez TAR & 462 & $(50,7)$ \\
2 INTR+1 INNTR & 251 & $(54,3)$ \\
2 INTR+1 IP & 137 & $(29,7)$ \\
INTR & 24 & $(5,2)$ \\
1 ó 2 INTR & 50 & $(10,8)$ \\
Fallecido & 189 & $(20,7)$ \\
\hline
\end{tabular}

*. dato consignado en 100 pacientes.

INTR: Inhibidores nucleósidos de la transcriptasa reversa. INNTR: Inhibidores no nucleósidos de la transcriptasa reversa. IP: Inhibidores de la proteasa.

Tabla 4. Análisis univariado de factores asociados a enfermedad tuberculosa

\begin{tabular}{|lccr|}
\hline Variable & $\begin{array}{c}\text { Enfermedad TBC } \\
(\mathrm{n}=62)\end{array}$ & $\begin{array}{c}\text { Sin enfermedad TBC } \\
(\mathrm{n}=850)\end{array}$ & $\begin{array}{r}\text { Valor } \\
\mathrm{p}\end{array}$ \\
\hline Edad (años), promedio $\pm \mathrm{DS}$ & $40,6 \pm 10,2$ & $37,6 \pm 11,1$ & 0,039 \\
Sexo femenino & $8,1 \%$ & $17,2 \%$ & 0,092 \\
Fallecido & $41,9 \%$ & $19,2 \%$ & $<0,001$ \\
CD4 basal $\left(\mathrm{cel} / \mathrm{mm}^{3}\right)$ & $143 \pm 148$ & $262 \pm 223$ & $<0,001$ \\
Log CV basal $(\mathrm{copias} / \mathrm{ml})$ & $4,92 \pm 0,95$ & $4,65 \pm 0,96$ & 0,033 \\
Reciben alguna vez TAR & $66,1 \%$ & $49,8 \%$ & 0,019 \\
\hline
\end{tabular}

Tabla 5. Prevalencia de enfermedad T BC en individuos con infección por VIH según zona geográfica y O R de enfermedad T BC por zona respecto a Arica, ajustado por edad y sexo

\begin{tabular}{|lccc|}
\hline Zona geográfica & $\mathrm{n}$ & Prevalencia (\%) & OR ajustado (IC 95\%) \\
\hline Arica & 144 & 5,5 & 1,00 \\
Valparaíso/San Antonio & 221 & 7,1 & $1,26(0,76-2,08)$ \\
Metropolitana Sur-Oriente & 360 & 3,9 & $0,67(0,38-1,18)$ \\
Concepción/Arauco & 187 & 11,9 & $2,42(1,50-3,90)$ \\
Total & 912 & 6,2 & - \\
\hline
\end{tabular}


Concepción/Arauco respecto a Arica se mantuvo incluso posterior al ajuste según edad $\mathrm{y}$ sexo. Por otra parte, al comparar las tasas de TBC con las de la población general correspondiente a las cuatro zonas evaluadas, se observa un paralelismo entre la tendencia de la tasa en la población general y aquellas observadas en los individuos VIH positivos, con excepción de Arica (Figura 1).
Impacto de la TBC en la mortalidad por VIH/SIDA. La enfermedad TBC aparece registrada como causa de muerte en $7,4 \%$ de los pacientes fallecidos. Para determinar si existió impacto de la enfermedad TBC sobre la mortalidad de PVVIH se realizó un análisis univariado que identificó una asociación estadísticamente significativa de mortalidad con enfermedad TBC, género masculino, mayor edad, menor CD4, mayor CV y no uso de TAR (OR crudo, Tabla 6). Sin

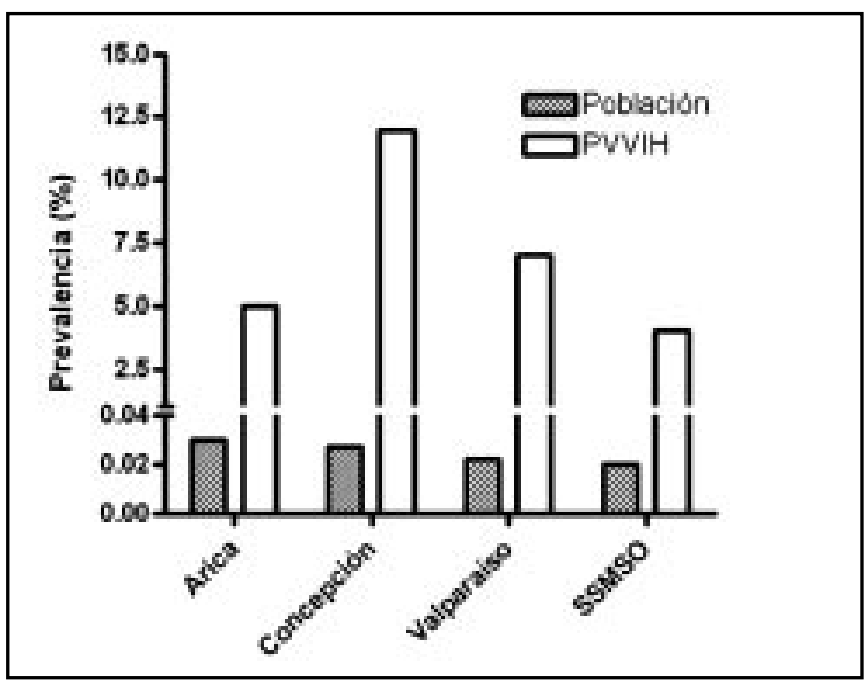

Figura 1. Prevalencia de TBC poblacional y en individuos con infección por VIH según Servicio de Salud.

Tabla 6. Análisis univariado (OR crudo) y multivariado (OR ajustado) de factores asociados a mortalidad de sujetos con infección por VIH de los 4 centros de atención incluidos

\begin{tabular}{|lccccc|}
\hline Variable & $\begin{array}{c}\text { Vivos } \\
(\mathrm{n}=723)\end{array}$ & $\begin{array}{c}\text { Fallecidos } \\
(\mathrm{n}=189)\end{array}$ & $\begin{array}{c}\text { OR Crudo } \\
(\text { IC 95\%) }\end{array}$ & $\begin{array}{c}\text { OR Ajustado } \\
(\text { IC } 95 \%) *\end{array}$ & Valor $\mathrm{p}$ \\
\hline $\begin{array}{l}\text { Edad promedio } \\
\text { (años) }\end{array}$ & $36,3 \pm 10,6$ & $43,5 \pm 11,0$ & $(1,043-1,075)$ & $(1,013-1,067)$ & 0,003 \\
Sexo femenino & 130 & 21 & 0,570 & 0,464 & \\
& $(18 \%)$ & $(11,1 \%)$ & $(0,349-0,932)$ & $(0,173-1,246)$ & 0,127 \\
CD4 basal & & & 0,995 & 0,993 & \\
$\left(\right.$ cel/mm $\left.{ }^{3}\right)$ & $279,9 \pm 222$ & $130,4 \pm 166$ & $(0,994-0,997)$ & $(0,991-0,996)$ & $<0,001$ \\
LOG CV basal & & & 2,047 & 1,513 & \\
(copias/ml) & $4,59 \pm 0,96$ & $5,14 \pm 0,82$ & $(1,541-2,719)$ & $(1,076-2,127)$ & 0,017 \\
Uso de TAR & 406 & 58 & 0,809 & 0,163 & \\
$(\%)$ & $(56,2 \%)$ & $(30,7 \%)$ & $(0,755-0,866)$ & $(0,085-0,312)$ & $<0,001$ \\
Enfermedad & 36 & 26 & 3,044 & 2,177 & \\
TBC (\%) & $(5 \%)$ & $(13,8 \%)$ & $(1,787-5,185)$ & $(0,913-5,192)$ & 0,079 \\
\hline
\end{tabular}

*Ajustado por las demás variables tabuladas mediante modelo de regresión logística. 
embargo, al ajustar usando modelos de regresión logística, las variables que se mantuvieron asociadas en forma significativa con mortalidad fueron el menor recuento de CD4, mayor edad, mayor CV y menor uso de TAR en algún momento de su seguimiento (OR ajustado, Tabla 6).

Infección latente TBC. De los 912 individuos VIH positivos, 265 (29,1\%) registraban examen de PPD al ingreso al programa VIH, con diferencias importantes de solicitud entre los diferentes centros (desde 5,9\% en Valparaíso/San Antonio hasta 82,1\% en Arica). El examen resultó positivo en 17 casos, lo que permite estimar la frecuencia de infección latente TBC en 6,4\%.

En 10 pacientes se registró uso adecuado de isoniacida y en ninguno de ellos se diagnosticó enfermedad tuberculosa.

\section{DisCUSIÓN}

La prevalencia de TBC en la muestra de individuos VIH positivos incluidos en este estudio es más de 300 veces superior a la tasa de morbilidad TBC de la población general. Esto confirma datos nacionales ${ }^{6} \mathrm{y}$ cifras internacionales que describen la alarmante frecuencia de TBC entre las $\mathrm{PVVIH}^{3}$. La mayor prevalencia de TBC en VIH se explica en parte por mayor susceptibilidad a reactivación de TBC latente con progresión a enfermedad ${ }^{11}$, aunque se ha descrito que hasta en un tercio de los casos sería atribuible a infección primaria por TBC con progresión rápida de enfermedad ${ }^{12}$.

De los resultados presentados, podemos caracterizar que la TBC en PVVIH en Chile es más frecuente a mayor edad, lo cual podría explicarse por diferencias en la frecuencia de TBC latente con el grupo más joven, sumado a deterioro inmunitario relacionado con la edad. Otra característica fue la mayor prevalencia de TBC en sexo masculino, si bien esta diferencia no alcanzó significación estadística, estudios en población general chilena también han descrito mayor incidencia de TBC en hombres que en mujeres, con una razón de $1,75^{4}$. La prevalencia de TBC extrapulmonar $(33,9 \%)$ es levemente superior a la descrita en la población general $(24,8 \%)$, lo cual confirma el mayor riesgo de PVVIH de formas diseminadas en especial con bajos recuentos de CD4.

Debemos destacar que la proporción de TBC latente diagnosticada mediante PPD en la población en estudio $(6,4 \%)$ fue muy similar a la proporción de enfermedad TBC (6,2\%). Este resultado sugiere que PPD pudiera aún ser un método útil en el diagnóstico de infección TBC en VIH. Sin embargo, se debe ser cauto en este valor ya que la frecuencia de infección TBC latente puede estar subestimada por la baja solicitud de PPD y por la condición de anergia de PVVIH especialmente entre aquellos pacientes con recuentos de CD4 bajos ${ }^{13}$. Por otro lado, no se puede descartar influencia de vacunación BCG sobre el resultado de PPD. Es reconocido que el antecedente de vacunación puede resultar en PPD positivo, si bien su reactividad va disminuyendo con el tiempo, se recomienda que en su interpretación se considere la situación clínica del individuo, edad y la prevalencia local de $\mathrm{TBC}^{14}$.

Una aproximación más exacta al valor real de infección TBC latente sería de alta importancia en el diseño de estrategias de disminución de progresión a enfermedad TBC. Es reconocido el elevado riesgo de $\mathrm{PVVIH}$ de progresión a enfermedad TBC; Horsburgh describió que PVVIH en el rango de edad 36-45 años con PPD >15 mm, 10-14 mm y 5-9 mm, los riesgos de reactivación a lo largo de la vida son $40 \%$ (IC 95\%, 20-79), 33\% (15-68) y $24 \%$ (8-68), respectivamente ${ }^{15}$, lo cual confirma la necesidad de una intervención en los individuos con PPD $\geq 5 \mathrm{~mm}$.

Las intervenciones estudiadas para disminuir el riesgo de reactivación de TBC en PPVIH, demuestran el beneficio del tratamiento de la infección latente con el uso de fármacos anti$\mathrm{TBC}^{16-19}$. Woldehanna y cols ${ }^{19}$ demostraron que la terapia con cualquier droga antiTBC comparada con placebo se asocia a un menor riesgo de TBC activa ( $R R=0,64$ IC 95\% 0,51-0,81), especialmente en los sujetos con PPD positivo ( $R R=0,38$ IC 95\% 0,25-0,57). En cambio en los sujetos anérgicos no se ha logrado demostrar el beneficio del uso de terapia anti-TBC. En un estudio español, comparando diferentes esquemas de tratamiento de eventual infección TBC latente en PVVIH anérgicos no se logró evidenciar diferencia entre 3 regímenes (isoniacida, 
rifampicina/isoniacida y rifampicina/pirazinamida) versus no-tratamiento ${ }^{20}$. Estos resultados deben considerarse en la planificación de una intervención a nivel nacional ya que la frecuencia de pacientes VIH anérgicos en nuestro país podría estimarse elevada en vista que $51,2 \%$ de los pacientes de la cohorte chilena ingresan con recuentos de $\mathrm{CD} 4<100 \mathrm{cel} / \mathrm{mm}^{3}{ }^{6}$. Interesantemente se ha reportado reactividad al test de PPD en PVVIH al recuperar CD $4>100 \mathrm{cel} / \mathrm{mm}^{3}$ bajo $\mathrm{TAR}^{21}$. Basándose en estos datos se puede plantear que en PVVIH con PPD negativo en una primera evaluación, podría repetirse esta prueba al recuperar $\mathrm{CD} 4$ a fin de definir su condición de infección TBC latente y en estas condiciones ofrecer tratamiento a aquellos que se confirme TBC latente.

A pesar de los datos de la efectividad del uso de drogas antiTBC en infección latente, su implementación y cumplimiento en la práctica clínica cotidiana no es fácil ${ }^{22}$, existe riesgo de toxicidad hepática $^{23}$, neurotoxicidad periférica y recientemente se ha descrito posible riesgo de resistencia con uso de isoniacida en monoterapia ${ }^{24}$.

El paralelismo observado entre las frecuencias de TBC en la población de los Servicios de Salud y las PVVIH de cada centro, con la excepción de Arica, hacen planteable que pudiese existir un enfrentamiento diferente del problema respecto a los otros centros, tales como la mayor solicitud de PPD en Arica respecto a otros centros. Esto podría determinar un mayor uso de isoniacida en el tratamiento de infección TBC latente con la consecuente disminución de la enfermedad TBC. Son necesarios más estudios para explicar las razones específicas que llevan a este comportamiento diferente al esperado. En el presente estudio, el riesgo de muerte ajustado por edad, sexo y recuento de CD4 fue 1,7 veces mayor en los pacientes con TBC respecto a los sin TBC sin alcanzar significación estadística. Además de la mortalidad directamente atribuible a la enfermedad tuberculosa, se ha descrito que la infección tuberculosa podría determinar una progresión más rápida del deterioro inmunológico asociado a la infección $\mathrm{VIH}^{25}$. Por otra parte, TBC activa puede retrasar el inicio de terapia antirretroviral por el riesgo de interacciones farmacológicas, provocando un mayor período de inmunosupresión en estos pacientes, y este factor también podría explicar la mayor mortalidad encontrada en este grupo.

Respecto a la observación de que los pacientes con enfermedad TBC habían recibido TAR más frecuentemente, esto incluye pacientes que inician TAR por TBC como aquellos casos eventuales que corresponden a reconstitución inmune. Si el inicio de TAR modifica la progresión de infección a enfermedad TBC, no puede ser definido a través de los datos presentados. Es planteable que en la medida que se dispone de TAR altamente eficientes los periodos de inmunodeficiencia serán más breves con la eventual disminución del riesgo de progresión a enfermedad TBC. Sin embargo, dado que no se conoce con exactitud la duración del beneficio de la terapia se podría plantear que, si bien el riesgo de enfermedad TBC puede disminuir en una primera etapa en aquellos pacientes con respuesta viroinmunológica adecuada, desconocemos qué ocurriría en aquellos PVVIH que presentan durante su seguimiento una falla inmunológica respecto al riesgo de progresión a enfermedad TBC.

En resumen, la enfermedad TBC en PVVIH en Chile es 300 veces más frecuente que en la población general de los Servicios de Salud incluidos en este estudio y tiende a seguir la tendencia de la TBC poblacional en cada zona geográfica, con excepción de Arica. Esta diferencia podría ser explicada por una diferente aproximación al problema TBC del centro de salud Arica. Se requiere determinar si las normas actuales ministeriales son las más adecuadas del punto de vista de efectividad, aplicabilidad y costos.

\section{Agradecimientos}

Los autores agradecen la disposición de la Comisión Nacional del SIDA y a su Coordinadora Ejecutiva, Sra. Edith Ortiz, del Ministerio de Salud, para aportar la información oficial sobre VIH/ SIDA. También agradecen al doctor Carlos Beltrán por sus valiosos comentarios al manuscrito. 


\section{REFERENCIAS}

1. World Health Organisation, Geneva. Global Tuberculosis database. In: http://www.who.int/mediacentre/factsheets/fs104. (Consultado el 20 de agosto de 2007).

2. Joint United Nations Programme on HIV/AIDS (UNAIDS) and World Health Organization (WHO); AIDS Epidemic Update: Special Report on HIV/AIDS, december 2006

3. Corbett EL, Watt CJ, Walker N, Maher D, Wimams BG, Raviglone MC ET aL. The growing burden of tuberculosis: global trends and interactions with the HIV epidemic. Arch Intern Med 2003; 163: 1009-21.

4. Ministerio de Salud. División de Prevención y Control de Enfermedades. Programa Nacional de Control de la Tuberculosis. Manual de Organización y Normas Técnicas, 2005. Disponible en URL: http:/ /www.minsal.cl/ici/manual_organizacion_normas_tecnicas_control_tbc.pdf. (Consultado el $20 \mathrm{de}$ agosto de 2007).

5. Ministerio de Salud. Departamento de Epidemiología. Evolución del VIH-SIDA, Chile 1986-2005, octubre 2006.

6. Wolff M, Beltrán C, Vásquez P, Ayala M, Valenzueia M, Berríos G, Arredondo A. The Chilean AIDS Cohort A model for evaluating the impact of an expanded access program to antiretroviral therapy in a middle-income country - Organization and preliminary results. J Acquir Immune Defic Syndr 2005; 40: 551-7.

7. KISH L. (1965). Survey Sampling. John Wiley and Sons, New York, USA.

8. Levy P, S. LemEShow. (1991). Sampling of populations. $2^{\text {nd }}$ Ed. John Wiley and Sons, New York, USA.

9. Hosmer DW, Lemeshow S. Applied logistic regression. Wiley 2000.

10. R Development Core Team (2006). R: A language and environment for statistical computing. $\mathrm{R}$ Foundation for Statistical Computing, Vienna, Austria. ISBN 3-900051-07-0, URL http://www.Rproject.org. (Consultado el 20 de agosto de 2007).

11. Girardi E, Raviglione MC, Antonucci G, GodfreyFausset P, IPpolto G. Impact of the HIV epidemic on the spread of other diseases: the case of tuberculosis. AIDS 2000; 14 (Suppl 3): S47-56

12. Center for Disease Control and Prevention. Treatment of tuberculosis. MMWR 2003; 52(RR-11).
13. Gram NM, Neison KE, Solomon L, Bonds M, Rizzo RT, Scavotto J ET AL. Prevalence of tuberculin positivity and skin test anergy in HIV-1 seropositive and seronegative intravenous drug users. JAMA 1992; 267: 369-73.

14. Tissot F, Zanetti G, Franciol P, Zelieweger JP, Zysset F. Influence of Bacille Calmette-Guérin vaccination on size of tuberculina skin test reaction: To what size? Clin Infect Dis 2005; 40: 211-7.

15. HorsBuRgh CR JR. Priorities for the treatment of latent tuberculosis infection in the United States. N Engl J Med 2004; 350: 2060-7

16. Wilkinson D, SQuire SB, Garner P. Effect of preventive treatment for tuberculosis in adults infected with HIV: systematic review of randomized placebo controlled trials. BMJ 1998; 317: 625-9.

17. HavilR DV, Barnes PF. Tuberculosis in patients with human immunodeficiency virus infection. $\mathrm{N}$ Engl J Med 1999; 340: 367-73.

18. Scholten JN, Driver CR, Munsiff SS, Kaye K, Rubino MA, Gourevitch MN ET AL. Effectiveness of isoniazid treatment for latent tuberculosis infection among human immunodeficiency virus (HIV)infected and HIV-uninfected injection drug users in methadone programs. Clin Infect Dis 2003; 37: 1686-92.

19. WoLdehanna S, Volmink J. Treatment of latent tuberculosis infection in HIV infected persons (Cochrane Review). In: The Cochrane Library, Issue 3, 2004. Chichester, UK: John Wiley \& Son, Ltd.

20. Rivero A, López-Cortés L, CastiLo R, Lozano F, García MA, Diez $F$ et aL. A randomized trial of three regimos to prevent tuberculosis in HIVinfected patients with anergy. Enferm Infecc Microbiol Clin 2003; 21: 287-92.

21. Fisk T, Hon H-M, Lennox J, Reyn CF, Horsburgh JR CR. Detection of latent tuberculosis among HIV-infected patients after initiation of Highly active antiretroviral therapy. AIDS 2003; 17: 1102-4.

22. Antonucci G, Girardi E, Raviglone M, Vanacore $P$, Angarano G, Chiriano A et al. Guidelines of tuberculosis preventive therapy for HIV-infected persons: a prospective, multicentre study. Eur Respir J 2001; 18: 369-75.

23. Gordin FM, Cohn DL, Matts JP, Chaisson RE, O’BRIEN RJ ET AL. Hepatotoxicity of rifampin and 
pyrazinamide in the treatment of latent tuberculosis infection in HIV-infected persons: is it different than in HIV-uninfected persons? Clin Infect Dis 2004; 39: 561-5.

24. Balcelds ME, Thomas SL, Godfrey- Faussett P, Grant $A D$. Isoniazid preventive therapy and risk for resistant tuberculosis. Emerg Infect Dis 2006; 12: 744-51.

25. Whaten C, Horsburgh CR, Hom D, Lahart C, SIMBERKOFF M, EUNER J. Accelerated course of human immunodeficiency virus infection after tuberculosis. Am J Respir Crit Care Med 1995; 151: 129-35. 